\title{
The periodontal environment of canine teeth
}

\author{
Olivier SOREL, Dominique GLEZ
}

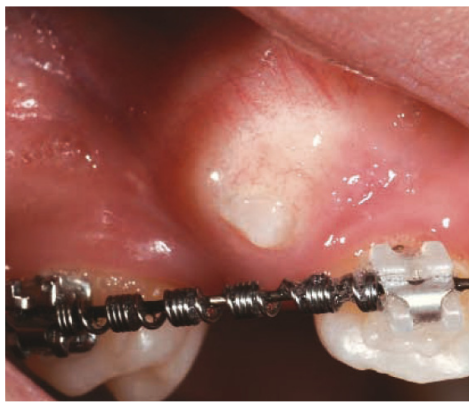

\section{ABSTRACT}

The periodontium plays a predominant role in the healthy stability of the dentition in general and of canine teeth in particular. After reviewing the make-up of healthy periodontal tissue and evaluating the situations where it is at risk, we describe different techniques of muco-gingival surgery that are applicable to canines.

We then illustrate the integration of periodontal data in treatment planning for orthodontic treatment of impacted canine teeth with clinical cases where our therapeutic choices have a certain original quality.

\section{KEYWORDS}

Canine

Periodontium

Muco-gingival surgery. 


\section{1 - INTRODUCTION}

The periodontal environment plays a capital role in the health of the dentition and especially of the canines, which are located at what might be called the crossroads of the arches. Of all the teeth, canines are the ones with the longest roots. They are situated between the anterior, incisal, and posterior, premolar-molar sectors of the arches. And they occupy the point where the change of arch curvature is maximal. The canine bulge, an important aspect of the tooth's architecture, exposes the buccal gingiva investing it to pressures exerted by cheek musculature, which explains the fragility that characterizes it.

\section{2 - THE PERIODONTIUM}

\section{2 - 1 - General observations}

The periodontium can be thought of as divisible into two sectors, the superficial layer, the gingiva, the only area available for clinical observation, and the deep periodontal layer.

\section{2 - 1 - 1 - The superficial periodontal layer, the gingiva}

The gingiva is the portion of the buccal mucosa that covers the alveolar processes and enwraps the necks of teeth. It can be divided into three elements:

- The marginal gingiva, diverging from the teeth, defines the gingivodental sulcus, up to a depth of $2 \mathrm{~mm}$. The sulcus is limited by the epithelial attachment at the bottom of the sulcus;
We shall explain in an initial section the special characteristics of the periodontium investing the canines, the way it is set in place during the eruption of these teeth and their maturation. We shall describe the indications for and the procedures of muco-gingival surgery whose objective is to preserve the integrity of the gingival attachment. Then we shall describe the individual features of actions needed to optimize the results of our orthodontic treatment efforts and the correct decisions we should make to guarantee the long-term stability of the dentitions that are entrusted to our care.

- The intermediate, or papillary, gingiva;

- The attached gingiva which adheres strongly to alveolar bone and to the root's cementum thanks to a network of fibers.

- In a clinical examination the orthodontist can evaluate the health of the gingiva:

- Contour: the gingival margin follows the contours of necks of teeth harmoniously in young patients. But as patients age increasing portions of root surfaces may become visible following gingival recession. This development should be limited and some authors call it abnormal but it does occur frequently and, especially, in the area of canines, especially in the maxillary arch;

- Amount and consistency: healthy gingiva is firm. Marginal gingiva should 
have tonicity and the sulcus should be a virtually closed space. In health the gingiva allows the examiner to send a probe through the sulcus but should close up immediately after the probe is removed with no bleeding or oozing liquid. The tonicity of this part of the gingiva protects the attached epithelial tissue. The border of the marginal gingiva should be thin, constituting a deflective architecture. The bulge of the tooth is another element in this protection.

- But in ageing and in cases of periodontal disease, the receding gingiva gets removed from the protective bulbous shape of teeth, making it more vulnerable;

- Color: healthy gingiva is rose colored, lighter than buccal mucosa. But it can assume a brownish coloration thanks to the melanoderme pigments of dark skinned people;

- Surface condition: healthy gingiva is usually smooth or somewhat dimpled like an orange peel. In diseased, edematous gingiva, the grainy surface persists.

- Histology of the gingiva

Gingiva is composed of conjunctive tissue fibers covered by a pavement type layer of stratified epithelium.

The epithelium of attached gingiva is keratinized or para-keritinized, unlike the tissue of the sulcus whose lack of tougher tissue covering permits the penetration of bacterial products from dental plaque into the conjunctive tissue, leading to inflammation.

The epithelium of the alveolar mucosa is fine and non keratinized ${ }^{8}$.

Palatal mucosa is hard and firmly attached to the underlying periosteum and, in the most part, has a keratinized epithelium, which makes it the preferred sites for oral surgeons to seek tissue for grafts.

\section{2 - 1 - 2 - The deep periodontium}

The deep periodontium is made up primarily of alveolar bone and cementum.

- The alveolar bone is in continuous relationship with basal bone, which, made up of an organic matrix and hydroxyapatite crystals, is structurally similar. But it exists only as a lodging site for teeth and, accordingly, possesses a more rapid turn over capacity than that of basal bone ${ }^{4}$.

It is difficult to assess the thickness of alveolar bone, especially in limited areas. But modern radiological techniques are giving us a better view of alveolar bone as we shall see later.

The principal function of the periodontal membrane, which is made up of non-specialized connective tissue, is to anchor the tooth in alveolar bone by means of a large quantity of collagenous inter-connecting fibers. It enjoys a proprioceptive sensibility that makes a considerable contribution to the sensitivity of the masticatory apparatus. About $0.1 \mathrm{~mm}$ thick, it possesses a reservoir of potential osteogenic and cementogenic cells and it is thanks to this structure and its capacities that orthodontic movement is possible.

- The cementum consists of nonvascularised connective tissue, tightly adherent to the root surface. In its embryonic origin it gives rise to the periodontal tissues. 


\section{2 - 2 - Development of the periodontal structures}

\section{2 - 2 - 1 - The setting up of Periodontal structures}

It is at the moment that teeth emerge into place that periodontal structures begin to take form, usually harmoniously.

The permanent canine teeth make their appearance during the second phase of the formation of the dentition when children are between 9 and 13 years of age, simultaneously with the arrival of puberty at a time when periodontal structures are most active $^{11}$.

The point at which a tooth emerges into the arch determines irrevocably the amount of keratinized tissue that will surround it. By making an early determination of where this point will be a dentist can predict its future periodontal environment (fig. 1 a to c).

An ideal emergence of a tooth, in the middle of the alveolar process will lead the tooth to have a periodontal architecture of type 1 in the classification of Maynard and Wilson ${ }^{9}$ or of type 4 in Korbendau and Guyomard's classification ${ }^{6}$.

But if the tooth appears instead somewhere in the alveolar mucosa, the functional epithelium located near the cemento-enamel junction will not be able to fuse with underlying keratinised gingival epithelium because there will be none available in that area. So the formation of a junction between attached conjunctive tissue and gingival tissue will be defective ${ }^{12}$ (fig. 2).

However, timely muco-gingival surgery before the tooth's eruption can correct the problem.
The presence of attached gingiva fosters good periodontal health ${ }^{13}$, which is why examining dentists should carefully evaluate the quality of keratinized gingiva and the height of attached gingiva in any examinations of children and adolescents, especially those undertaken before inception of orthodontic treatment and during it ${ }^{1}$.

\section{2 - 2 - 2 - Maturation of periodontal structures}

It is at the moment of a tooth's emergence through gingival tissue that the height of attached gingiva is determined. The muco-gingival line remains stable during the temporary dentition just as it does during the permanent dentition. In the temporary dentition the dimensions of the attached gingiva and the keratinized gingiva grow simultaneously following the growth of the alveolar processes. Then these tissues stabilize as the individual reaches adulthood $^{2,3}$.

The height of the attached gingiva in the permanent dentition of teen-agers is lower than it was in their temporary dentition but it increases progressively in stature as adolescents approach adulthood and in so doing reduces the depth of the gingival sulcus. It takes about ten years after the eruption of the permanent dentition for the average value of sulcular depth to equal that of adults ${ }^{14}$.

During this period, many adjustments of tissues and structures that modify a healthy periodontium take place. At the beginning of the eruption of teeth, the marginal gingiva is thick, double edged, flabby, and red, rarely spared from the presence of 


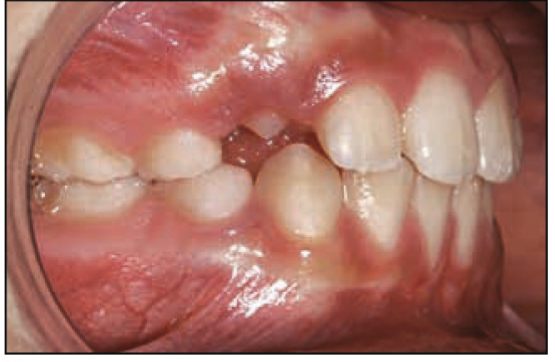

a

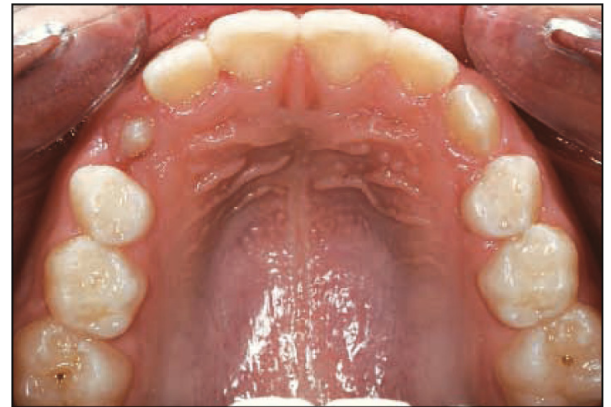

b

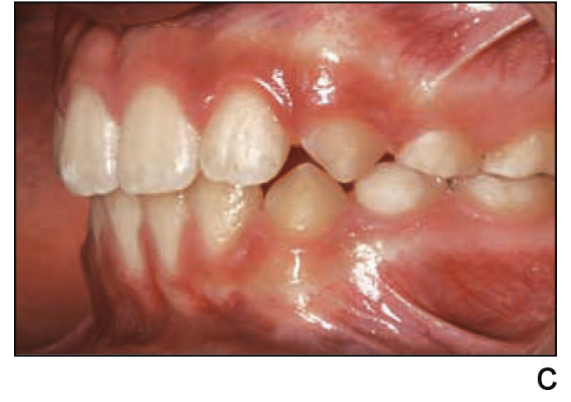

Figures 1 a to $c$

The upper right canine has just made its way through the mucosa in a favourable zone. Accordingly the attached gingiva will be arrayed harmoniously around it as will be the case with its mate on the left side of the arch. The point at which a tooth emerges determines, permanently, the amount of keratinized tissue that will invest it. At this early stage a dentist can make an accurate prediction about its future periodontal environment.

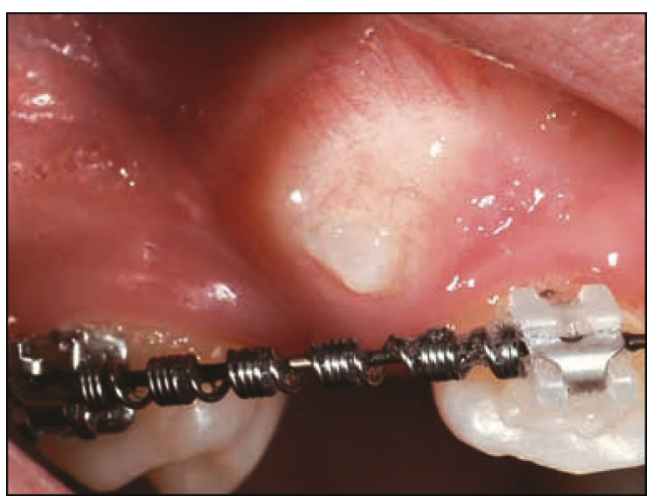

\section{Figure 2}

This maxillary canine has just pierced the mucosa in an unfavourable zone. Because the patent's parents had delayed in calling the periodontist for a check-up, it is now too late for an apically displaced flap to be laid back. The attached gingiva is already partially in position and the eruption of the canine's occlusal tip prevents the removal of sufficient tissue. inflammatory cells, but then, slowly, it matures ${ }^{7}$.

By acquiring a thorough understanding of the anatomic particularities of the gingiva of children and adolescents, dentists will be able to distinguish clinically normal situations from those that are pathological, to determine which conditions are at risk of worsening, and to envisage a comprehensive overview of the indications for muco-gingival surgery in order to prevent unfavourable developments or to begin orthodontic treatment under risky conditions.

\section{2 - 3 - Risky conditions}

\section{2 - 3 - 1 - Special anatomic situation}

The canine teeth are located in an intermediary zone in the arches between the incisal and the molar regions. R. Benoit described this situation with special clarity in the previous issue of this journal. The canines progressively integrate themselves into the functional schema that the first permanent molars had already established. It is in the canine areas that a change occurs in the curvature 
of the dental arches, which are generally elliptical in the homo sapiens species but are widely variable in shape both within and between different population groups. But, in all cases, canines assume a more or less buccal position that lends them the characteristic canine bulge, which also varies in intensity (fig. 3 a to c).

When this buccal placement is most marked, the tooth and its periodontium are subjected to the centripetal forces of the buccal envelope as well as to other exogenous pressures like tooth brushing. Clear evidence of dimorphism can be seen in these scanner images: buccally, cortical bone thickness is diminished and the height of the alveolar bone is less than it is palatally (fig. 4 a to c). The height and thickness of alveolar bone vary greatly from individual to individual and it is not rare to find instances of osseous fenestration in areas where the gingiva has a normal appearance (fig. 5 a to $\mathrm{c}$ ). This clinical situation, objectified by modern volumetric conical tomography, is one that practitioners should ponder and comprehend.

\section{2 - 3 - 2 Inflammation}

Gingivitis develops more slowly in children than it does in adults. But a brief episode of gingival disease occurs in $85 \%$ of 10 to 15 year-old patients when permanent teeth erupt as they are passing through puberty. Plaque is an essential component of the disease according to Timmerman ${ }^{15}$ but the gingival disease that it causes in young patients does not systematically worsen into periodontitis.

When patients with poorly aligned teeth fail to maintain good oral hygiene and at the same time have musculature that exerts powerful intraoral forces, these combined factors may lead to a loss of gingival attachment.

The risk of this type of damage is increased in the mouths of children with insufficient adherent gingiva. That is why it is especially important for them to maintain impeccable oral hygiene.

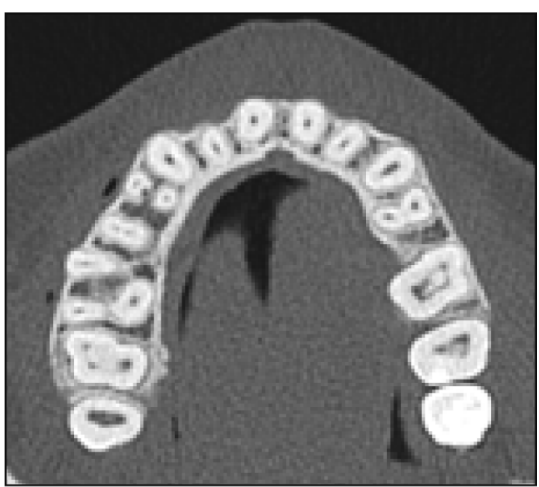

a

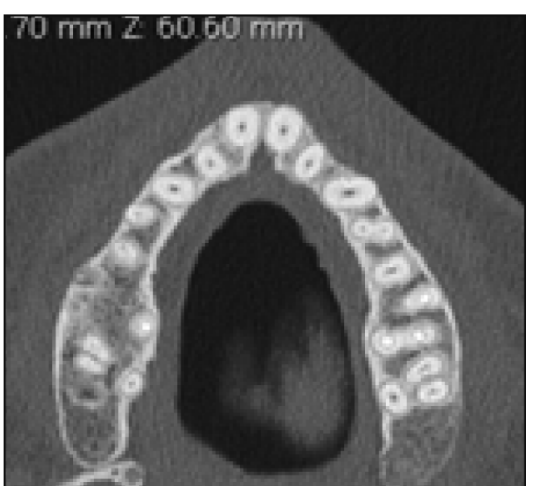

b

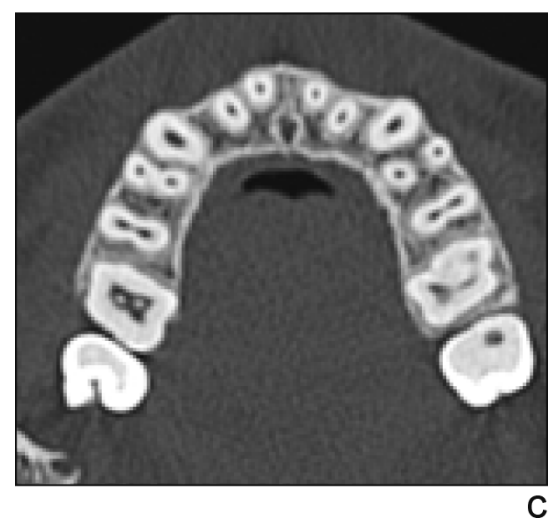

C

Figures 3 a to $c$

These scanner images show coronal maxillary sections of three patients that demonstrate different arch shapes: parabolic (a); V-shaped (b); and U-shaped (c). 


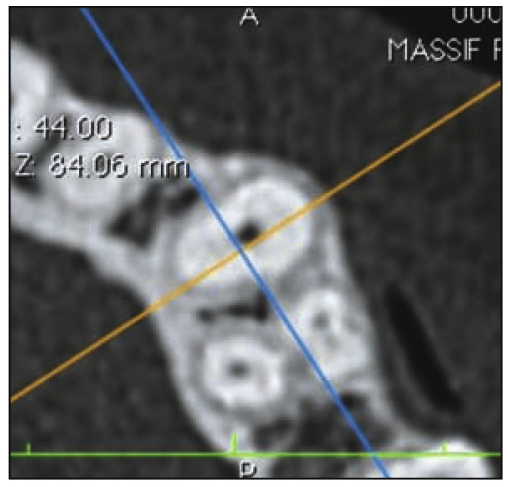

a

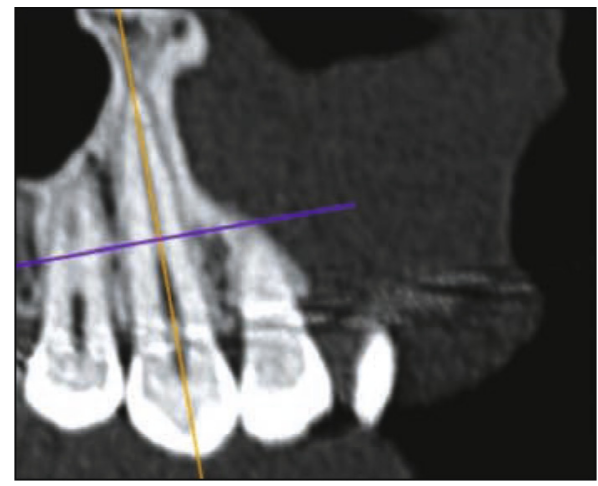

b

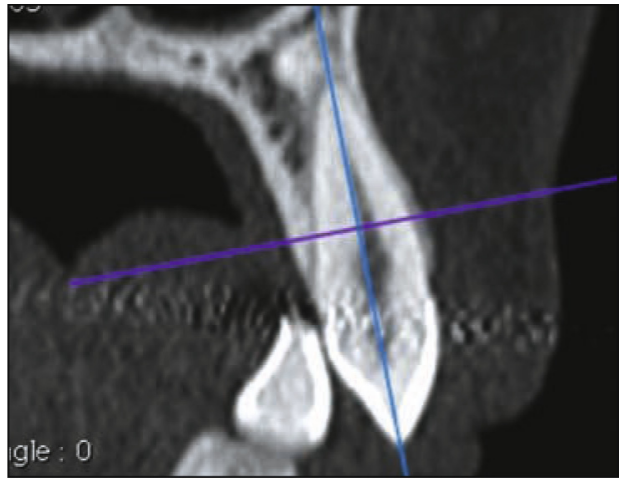

C

Figures 4 a to $c$

In volumetric films the practitioner can visualise a section of the tooth at any desired level. The coronal section (a) shows the relationship of the canine root with its environment. The plane of this section can be visualised in the other sections by means of the violet line. The axial section is para-sagittal (b). Its orientation can be followed using the blue line, which gives a good view of the septa between the teeth. The frontal axial view (c), with a yellow line for orientation with other images, gives a good view of buccal and palatal alveolar bone. This view shows the difference between the thickness of the buccal and palatal cortical plates in unusually stark contrast.

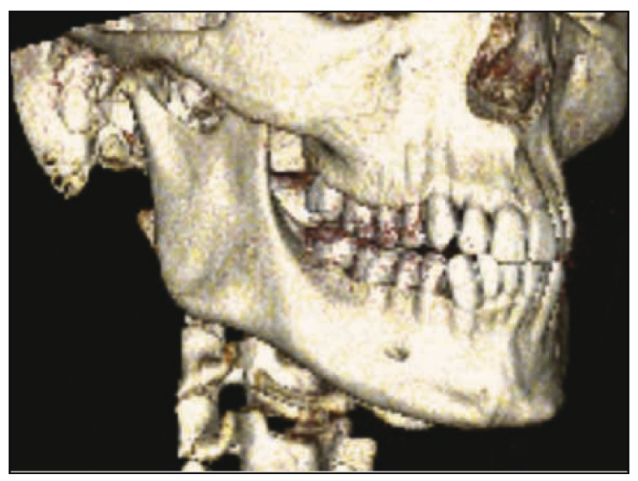

a

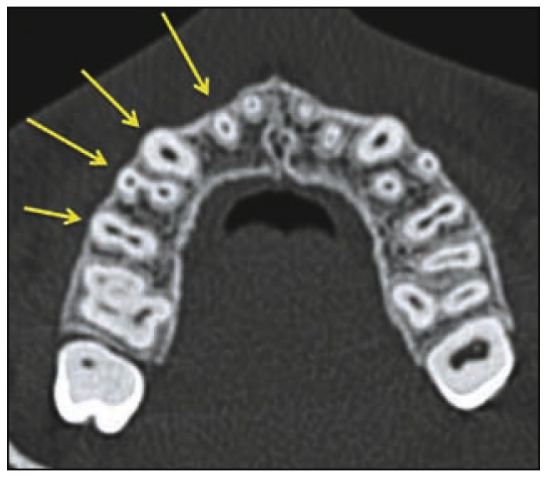

b

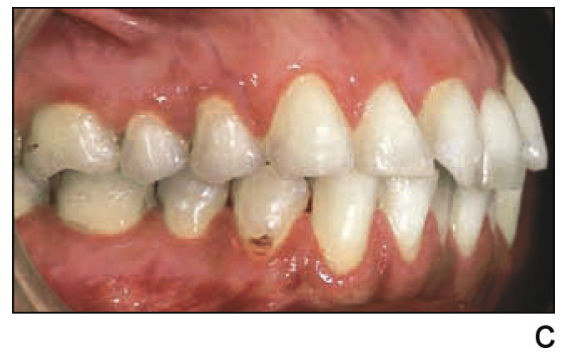

Figures 5 a to $c$

The scanner image of the arch (b) is placed between a skeletal reconstruction (a and an intraoral photograph, which gives no indication of the canine bulge large enough to lead to buccal cortical breakdowns that would be obvious on palpation. The yellow arrows in the X-ray show the buccal cortical fenestrations. But practitioners must be wary in interpreting such views, because the angulations of certain sections could make the problem appear worse than it actually is.

It is also important for orthodontists to evaluate all these factors when preparing a treatment plan and for them to vigilantly supervise home care during the course of treatment.
If, during orthodontic treatment, the periodontium shows an alteration in its integrity, in quality or quantity, lesions, whether or not provoked by mechano-therapy, will appear and the 
soft tissues will no longer follow along with the moving teeth ${ }^{5}$.

\section{2 - 3 - 3 - latrogenic trauma caused by patients}

Patients who brush their teeth with too much vigor may traumatise gingival tissue enough to cause recession. Children are often too impatient to learn how to brush their teeth properly and, sometimes decide that they can compensate for their failure to devote enough time to good oral care by using a hard brush too aggressively and too briefly. Adults, of course, can adopt the same delusion and brush so vigorously that the root surfaces of their teeth are abraded, denuded of gingival cover, and associated muscular tissue abused in a process of myolysis.

Some habits like nail biting, or onychopagy, or persistent chewing on a pencil or the stem of a pipe can also traumatize soft oral tissues. Intraoral "piercings" can be chronic gingival irritants sometimes leading to infections.

\section{3 - MUCO-GINGIVAL SURGERY}

Muco-gingival surgery can be used to correct the shape of superficial gingiva degraded by unfavourable anatomic conditions or by periodontal disease or simply in the context of overall soft tissue management. Operative techniques are capable not only of ameliorating the quality of visible tissues but also, as a general rule, of compensating for functional and aesthetic defects of the gingiva. It can also prevent the epithelial tissue of a pocket from creating a break of the muco-gingival border that would lead to a rupture of the attachment.

Muco-gingival surgical procedures can be categorized into two types, the additive, like grafts, that compensate for loss of substance, and the subtractive that correct unfavourable anatomic soft tissue bulk.

\section{3 - 1 - Grafts with pedicles}

\section{- Pedicle graft moved laterally}

This technique consists of displacing a partial thickness graft, contain-

ing the epithelium and a superficial portion of the superficial connective tissue, toward a neighbouring site where gingival recession has occurred.

\section{- Graft repositioned apically}

A partial thickness graft or one of the muco-periosteum is made in the course of a pre-prosthetic crown lengthening procedure or to encourage a child's tooth to emerge into a more favorable position than the one it was heading for. To begin the procedure, the oral surgeon makes a primary incision following the marginal gingival festoon exposing an internal bevel; next the surgeon makes an incision in the sulcus and a third horizontal cut designed to eliminate the epithelial border and expose the underlying connective tissue. Next two oblique incisions are made from one end of the site to the other thus liberating a trapezoidal flap with a large base that benefits from as generous a vascular supply as possible (fig. 6 a to $c$ and $7 \mathrm{a}$ and $\mathrm{b}$ ). 


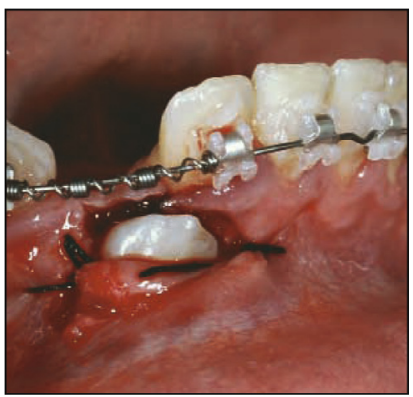

a

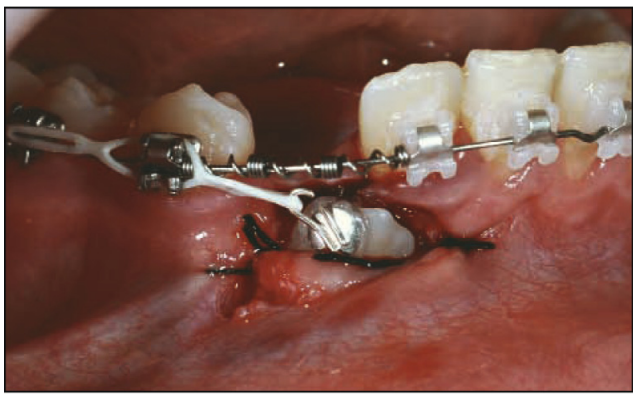

b

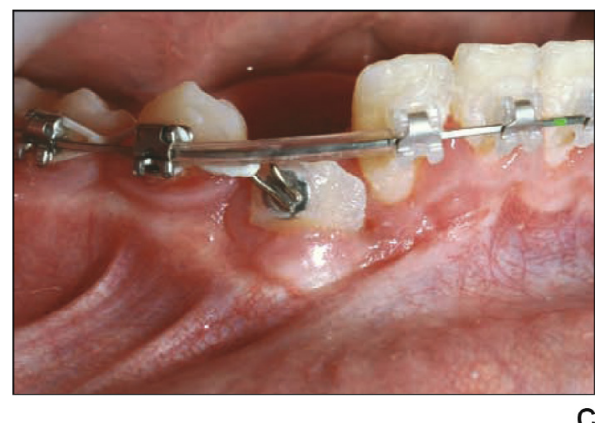

Figures 6 a to $c$

The intraoral photo (a) shows the uncovered canine with a repositioning of the attached gingiva. Traction can be seen in (b) applied to the unerupted tooth by means of a hook bonded near the tip of its cusp. After 4 months (c) the tooth can be seen progressing nicely towards its correct place in the arch with the attached gingiva in correct position.

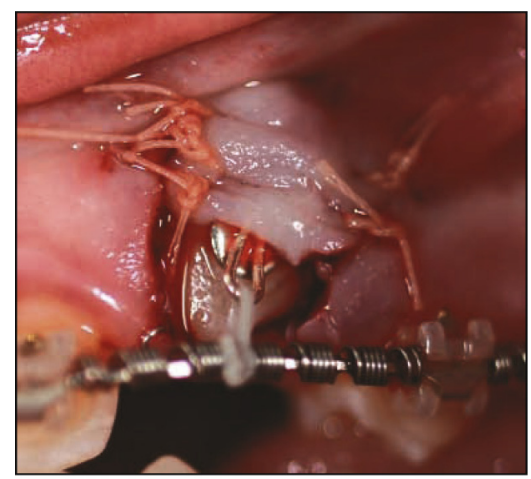

a

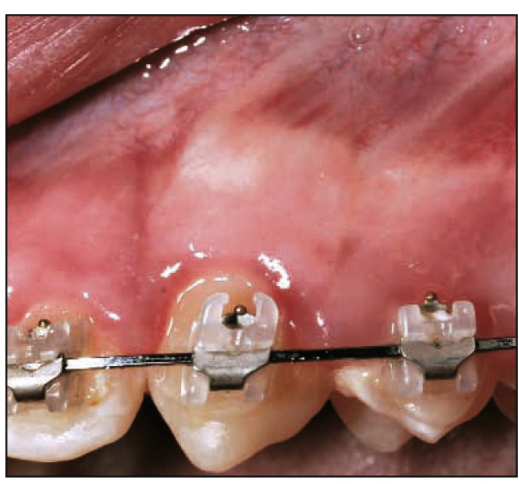

b

Figures $7 a$ and $b$

This canine was about to emerge in free gingiva. In intraoral photo (a) we see the apical displacement of an attached gingival flap. Photo (b) shows the orthodontic set-up that moved the tooth down into place bringing its attached gingiva along with it.

\section{3 - 2 - Free grafts}

\section{- The epithelial-conjunctive tissue graft}

(Fig. 8 a to $\mathrm{c}$ )

This technique of reinforcing superficial periodontal tissue has 3 operative stages.

- Preparation of the reception site. In order to give the graft as much nourishment as possible the surgeon extends the site as widely as possible, from one papilla to the other, removing epithelium from both and apically by means of bilateral incisions that liberate the graft at the base of defective area. The surgeon cleans the uncovered portion of the root, removing any bacterial toxins and finishes by remodeling the tooth's crown or root or both, especially if the root is too prominent. The root is also treated chemically to encourage connective tissue reattachment; 


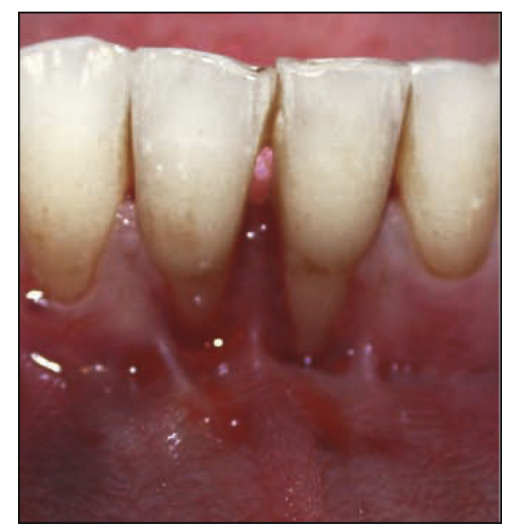

a

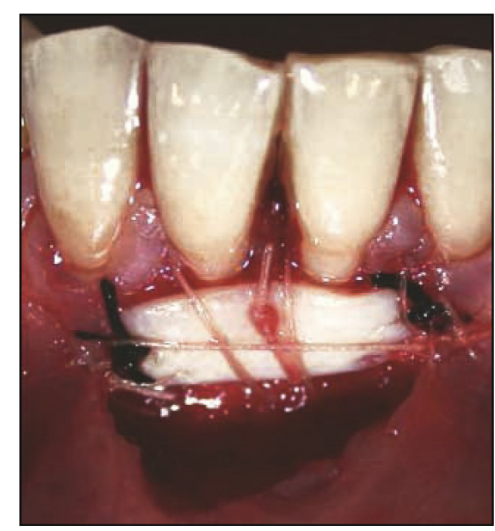

b

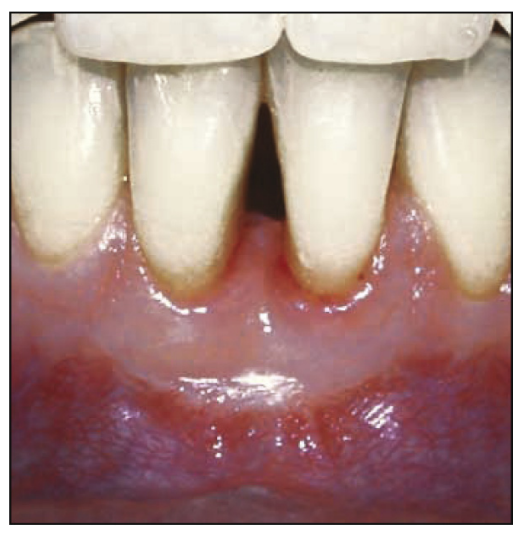

C

Figures 8 a to $c$

The intraoral photograph (a) shows marked gingival recession of the lower central incisors with traces of scar tissue. An epithelial-conjunctive tissue or free graft is sutured in position in photo (b). And, in (c) after healing, we can see the reinforced band of attached gingiva. The graft is well integrated but might be criticised for having given the teeth somewhat less than optimum coverage and for having a somewhat unpleasing bleached-out coloration.

- Taking a flap from another site. The surgeon usually dissects out fibromucosal palatal tissue from the region between the first premolar and the first molar to avoid a posterior vascular pedicle or from an edentulous area. The surgeon locates the epithelial surface by passage of a needle placed in the free portion of the graft and the fatty conjunctive tissue, which is excised. The surgeon assure cessation of palatal bleeding by pressure that can be supplied by a thermoformed plate that a patient may wear until the donor site has healed;

- Adaptation of the graft to the receptor site. The surgeon accomplishes this with sutures on the vertical borders, exerting compression, then places a continuous suspended suture joining the apical periosteum to the neck of the tooth; followed by more compression to extrude blood trapped under the graft thus encouraging more intimate contact with the surface of the newly recovered connective tissue.
Long term results are sometimes disappointing cosmetically with the graft giving a patched-in appearance with a "long" epithelial attachment despite the the phenomenon of exuberant attachment observed in the first few months after placement of the graft.

This type of procedure can be indicated as a prophylactic measure before prosthetic or orthodontic or to stop the progression of localised recession.

\section{- Deep conjunctive tissue graft}

(fig. 9 a to d)

The surgeon removes the connective tissue graft by dissecting out a section of fibro-mucosa from the palate which is then sutured over and left to heal. The surgeon prepares the reception site by laying back a partial thickness flap that will cover the graft thus insuring an adequate blood supply. This technique promises a high percentage rate of success and an aesthetic result far better than in the technique previously described. Now considered the treatment of choice in muco-gingival surgery, this 


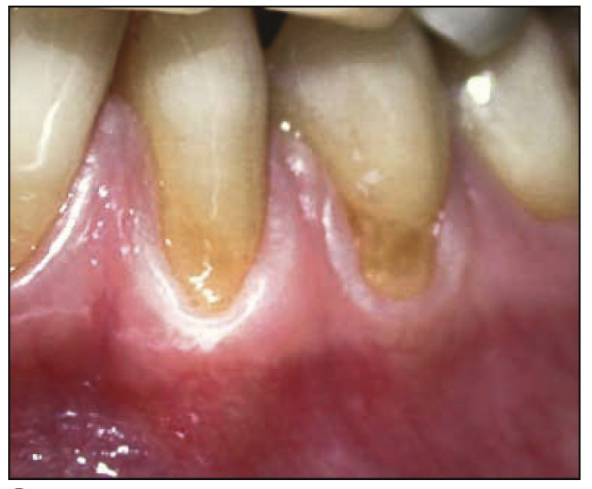

a

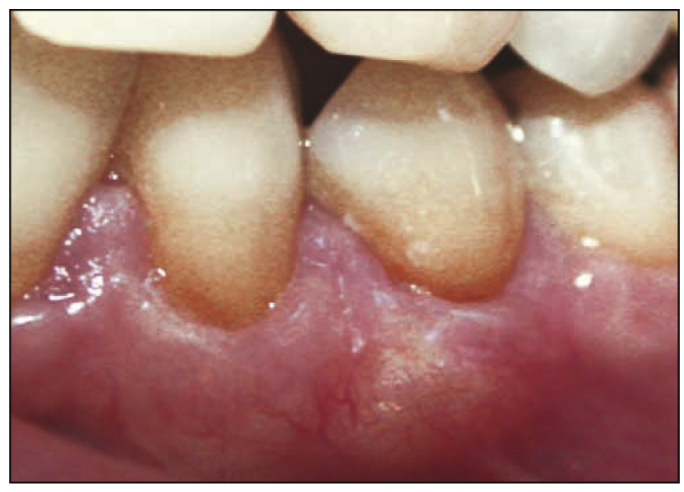

C

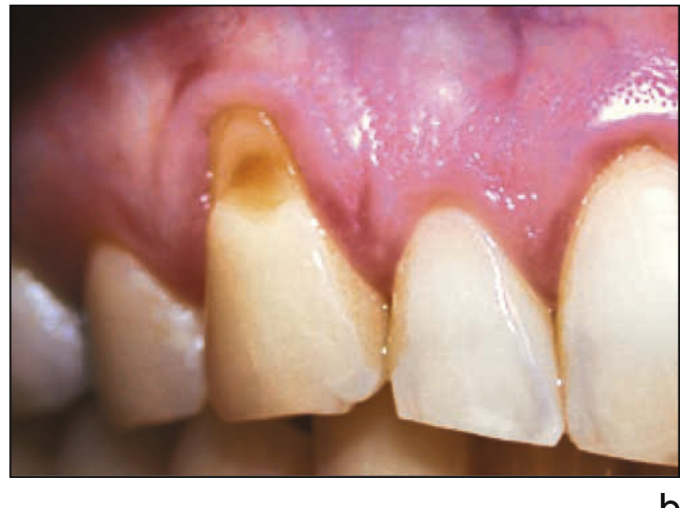

b

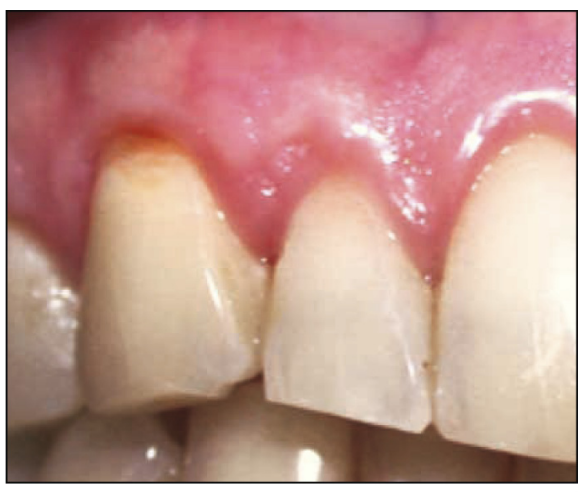

d

Figures 9 a to $d$

In the intraoral photographs (a and b) one can see gingival recession, a lowered position of gingival attachment, and marked myolysis of root dentine of several teeth with the brownish discoloration characteristic of this phenomenon. The risk of the situation worsening is high enough to contraindicate the initiation of orthodontic treatment. On photos (c and d) one can see how, after a deep connective tissue graft, the situation has returned to normal. The involved teeth now have a protective shield of keratinized attached gingiva which makes it possible for the orthodontist to begin mechano-therapy. NB. There are no pockets in the region where the recession was covered but there is a long epithelial junction.

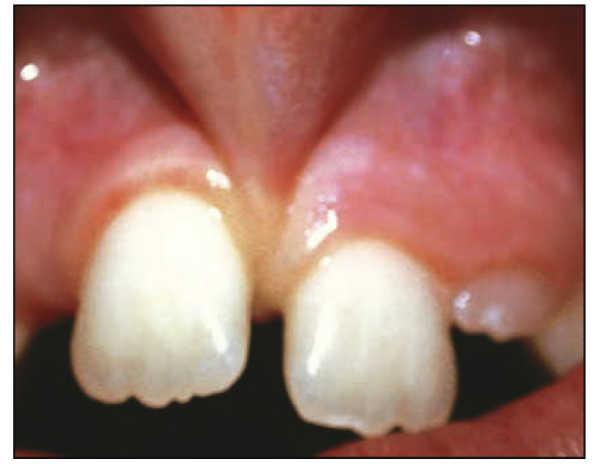

a

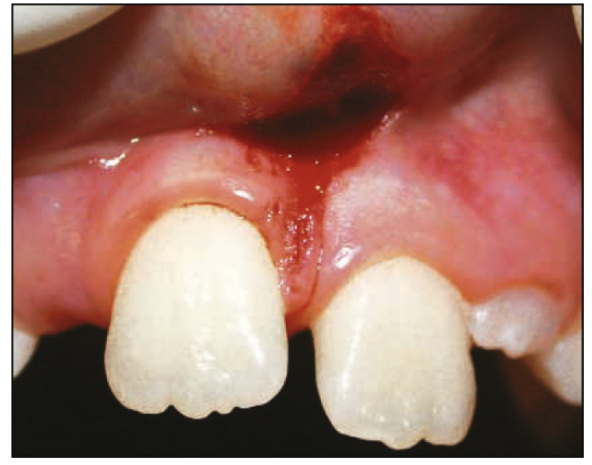

b
Figures $10 a$ and $b$

The original status of the bulky frenum between the central incisors can be seen on intraoral photograph (a). Photograph (b) shows the site after the frenum had been removed with a laser. 
procedure is often used in associated with guided tissue regeneration techniques, implantology, and before and during orthodontic treatment.

\section{3 - 3 - Techniques of tissue removal}

\section{- The frenectomy}

(fig. 10 a and b)
Frenectomies are now most frequently performed with lasers on tight and prominent buccally attached bands. After the incision is made in their intermediary zone, the fibers are pulled apart to detach them from their osseous insertion.

\section{4 - DECISIONS ABOUT CANINES IN ORTHODONTIC TREATMENT PLANS}

Canines play such a key role in the function of the dentition that dentists generally consider that extracting one of them for a child or a teen-ager would be as unthinkable as removing a central incisor. But for adults compromises required by very special clinical situations can sometimes impose just such an unlikely choice ${ }^{10}$.

In this chapter we are not dealing with the problem of bringing impacted cuspids into place in the arch, a procedure requiring the inter-disciplinary cooperation of oral surgeons, periodontists, and orthodontists that Van Beck has already described in another section of this issue.

We propose to discuss the atypical choices that we are sometimes called upon to make when an orthodontic treatment plan for an adult requires the extraction of teeth. The periodontal environment may influence our pragmatic diagnostic assessment in ways that will lead us to make highly inhabitual choices.

\section{4 - 1 - Making do without a canine!}

Extracting a canine means depriving the dentition of a tooth that has the largest single root in the arch. But the principle that requires us to select for therapeutic orthodontic extraction, especially in adults, those teeth that have the poorest prognosis sometimes requires that we do exactly that. Today, with our capability of creating removable bone-based anchorage we can establish treatment plans that include tooth movements heretofore difficult or impossible to achieve. For example, extracting a diseased second bicuspid instead of a healthy first bicuspid might formerly have posed insurmountable anchorage problems that can today be resolved with a mini-screw set in bone.

The cases that orthodontists treat without leaving canines in place are primarily those where the canines are missing to begin with or those with canines that have erupted into such a remote ectopic position that it is impossible to bring them into the arch.

But there are occasions when a canine has a poor prognosis and where its removal could so readily be incorporated into the proposed scheme of tooth movement that orthodontists choose to extract it.

In some cases where mesial movement of buccal segments is the primary cause of pronounced anterior 


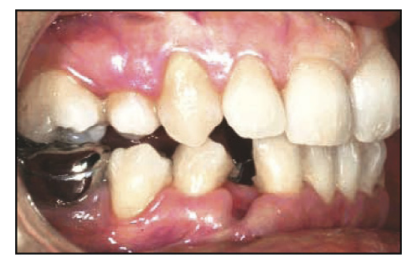

a

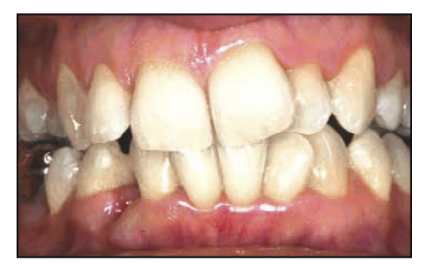

b

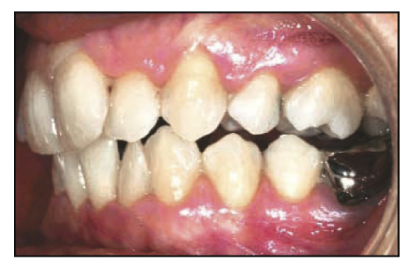

C
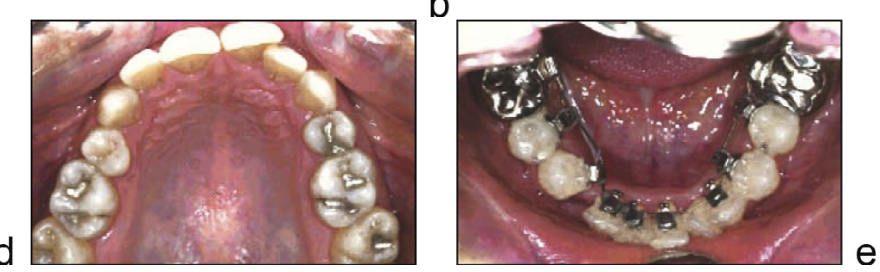

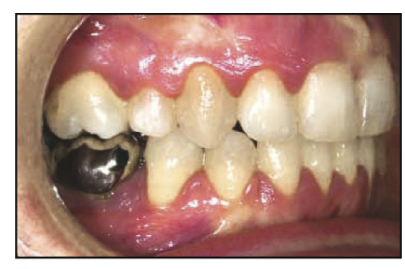

f

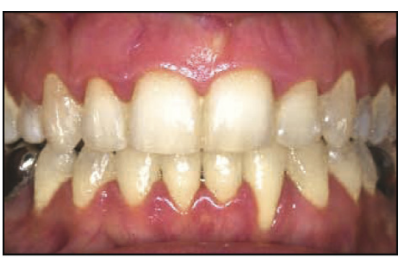

g

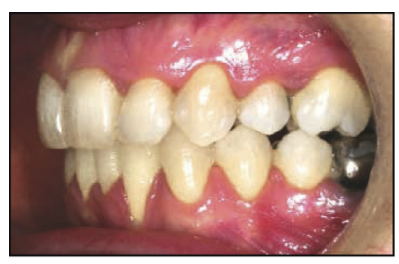

$\mathrm{h}$
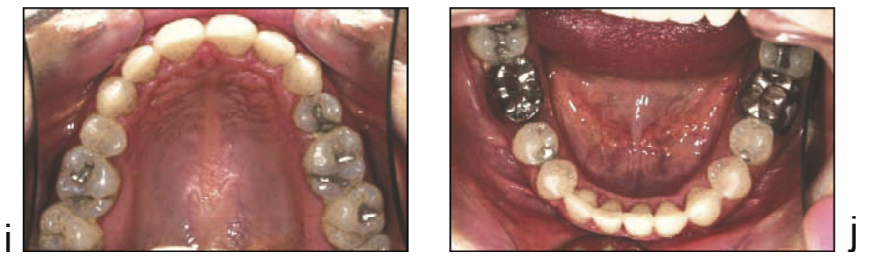

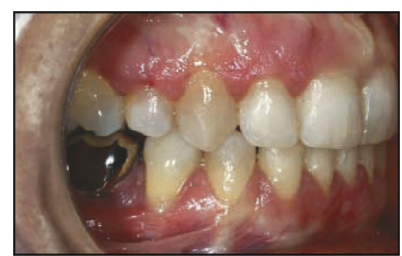

k

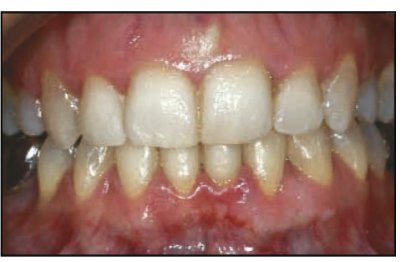

I

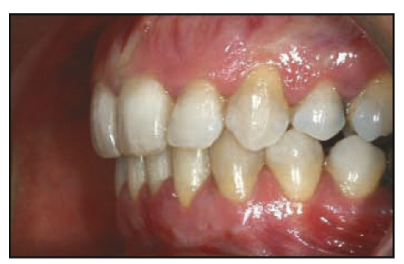

$\mathrm{m}$

n
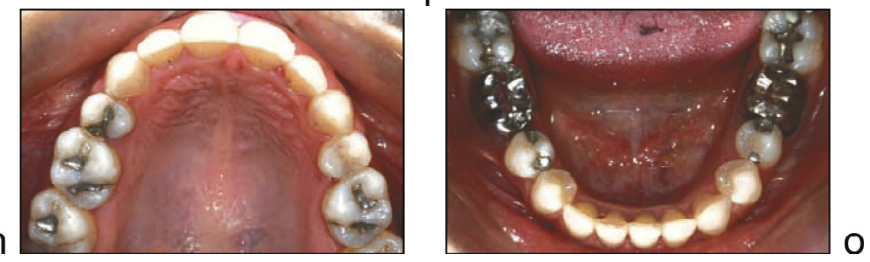

Figures 11 a to $o$

The intraoral photos (a to e) depict an initial situation of a dentition already treated with the extraction of two upper premolars in which maxillary arch is slightly crowded and the mandibular arch more severely crowded with 43 in a marked mesial position and suffering from severe gingival recession that put it at great risk. The dehiscence at the site of previously extracted first premolar was marked. At the end of treatment ( $f$ to $j$ ) the arches are harmoniously arranged and 44, with healthy periodontium, has replaced 33. It should be noted that the gingival recession on 32 will be corrected with a deep connective tissue graft. The intraoral post-retention photos ( $k$ to o) show that the arches have retained their harmonious forms. 
crowding, the buccal gingival covering of canines will be excessively thin and often already receding. Here the therapeutic choice confronting the orthodontist is simple:

- Either extract the first premolar and move the canine distally to provide room for alignment of the incisors, a plan that risks provoking further loss of the already fragile buccal gingiva during the distalization of the canine;

- or extract the canine itself, a plan that makes the needed space for incisal alignment immediately available, reducing the amount of required tooth movement and preserving a tooth with a healthy periodontium. Attaining satisfactory functional occlusion with this scheme is not difficult and it in no way detracts from the satisfactory aesthetic appearance of the dentition (fig. 11 a to o).

Following the same line of reasoning, orthodontists might consider extracting a lower lateral incisor in cases of Class II Division 2 malocclusions and moving the canine forward into its place thus automatically putting the upper canine into a Class I relationship.

\section{4 - 2 - Keeping a canine in a place not its usual position}

To illustrate this unusual situation we shall present two adult cases to describe the atypical choices an orthodontist might make.

The first patient consulted us about an upper lateral incisor that was forced into an unsightly labial position. (fig. 12 a to I). She had an asymmetrical occlusion, Angle Class II, subdivision left. While this patient had a slight skeletal imbalance it was not severe enough to require thoroughgoing orthodontic therapy. The upper midline, which had shifted, no longer matched the mandibular midline, which was centered in her face.

Because of the buccal gingival recession around 24 it seemed to us at first a good plan to extract it, then to move the buccal teeth forward partly into the space and to move 23 distally into the remaining space, thus making room for the lateral. However this plan would require considerable movement of the buccal teeth previously well integrated into the upper arch and occluding well with the lower arch into a zone of questionable periodontal integrity. The weakest tooth in the ensemble was, unquestionably, the thin-rooted lateral incisor, while the canine had a relatively short root.

After mature reflection, it occurred to us that extracting the feeble lateral and moving the canine into its place could solve the problem with a minimum of orthodontic tooth movement. We tested this unusual scheme with a set-up and were encouraged by the way it seemed to work, with its benefits of preserving the teeth with the strongest potential promising a short treatment time that turned out to be 10 months. The patient had no TMJ problems before treatment and none after. The reshaping of the upper left cuspid allowed it to take its correct and harmonious position in the patient's smile, which was her primary objective in seeking treatment.

Similar re-contouring and repositioning of canine teeth have become common practices in treating patients whose upper lateral incisors are congenitally absent and, more rarely, to 


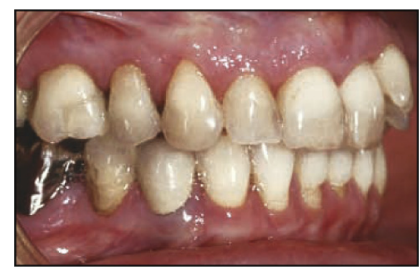

a

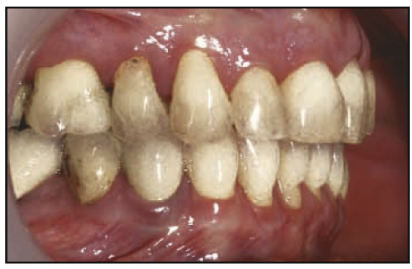

d

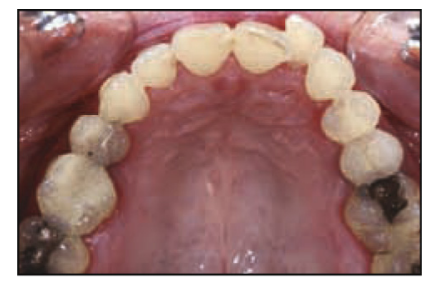

g

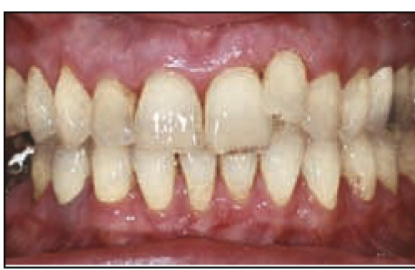

b

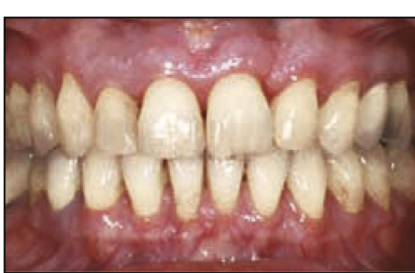

e

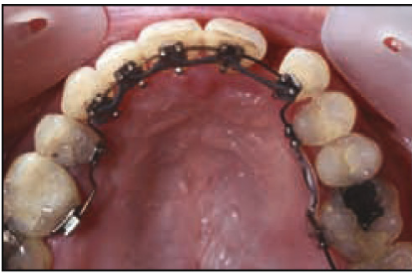

h

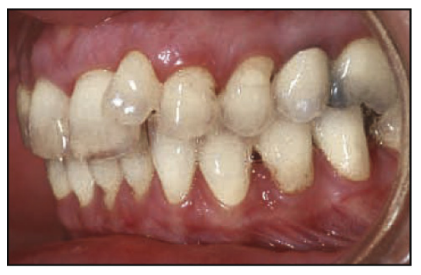

C

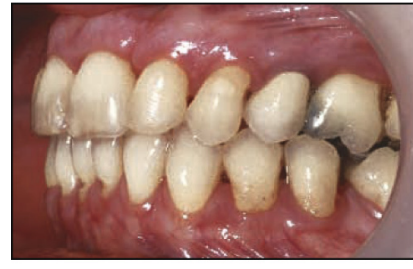

f

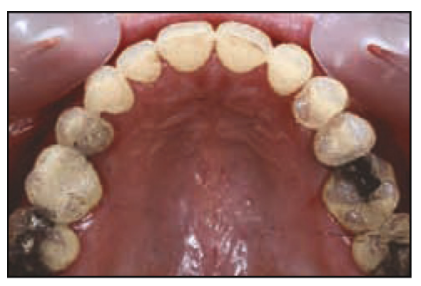

i

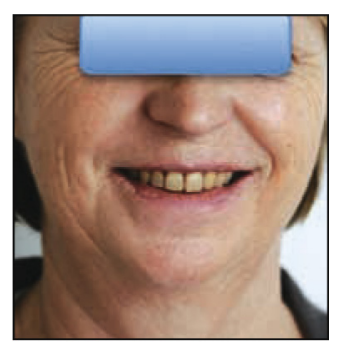

j

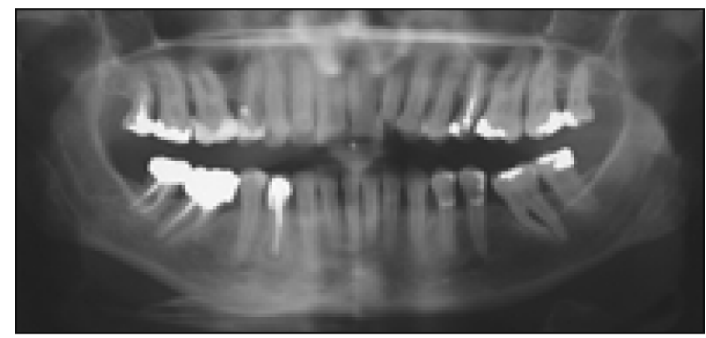

k

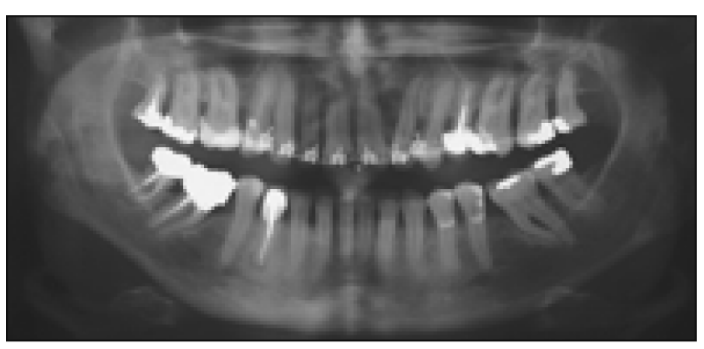

Figures 12 a to I

Intraoral photos of a 56 year-old female who had a Class II subdivision left malocclusion. In the photo of the right side, the Class I position of the canine and, because of the absence of an upper bicuspid, a Class II position of the first molar can be seen. In the frontal view the midline deviation is clear. A clinical exam showed that it was the mandibular midline that was centered in the face. On the left side both the molars and canines are in Class II relationship. In our opinion three considerations justified our choice of the upper left lateral for extraction: it was the tooth with the most feeble root, its removal would shorten the distances we would have to move other teeth, and its removal would simplify our re-centering the deviated upper midline. By reshaping the upper left canine to make it look like a lateral we were able to achieve an aesthetic, harmonious, and equilibrated result. The before, during, and after occlusal views ( $g$ to i) show the treatment benefits. In photo (j) the patient's harmonious smile can be seen and the panoramic films ( $\mathrm{k}$ and I) demonstrate how the accentuated angulation of the upper left cuspid helped the dentist to reshape it into the form of a lateral incisor. 


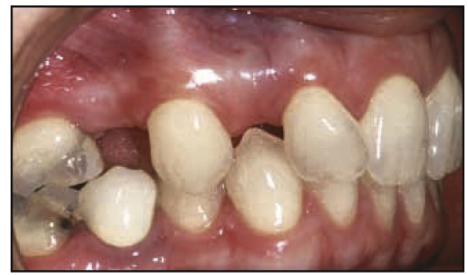

a

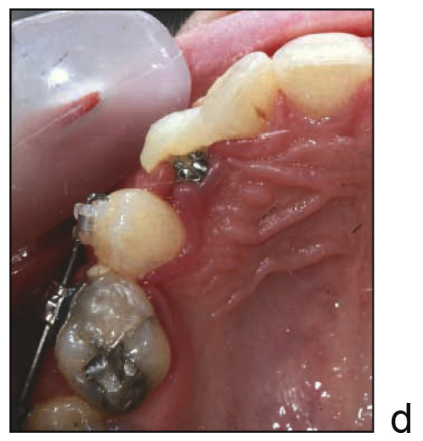

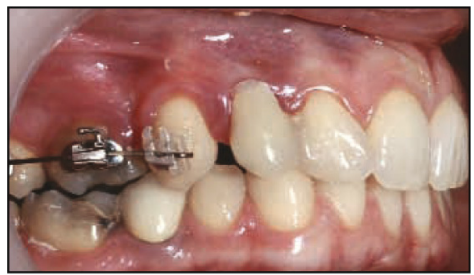

b

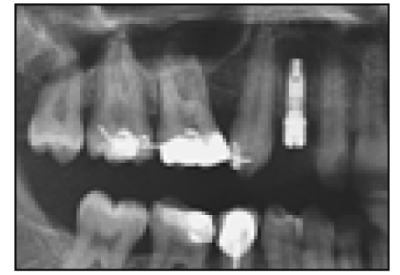

C

Figures 13 a to $d$

The initial situation depicted in (a) was one in which the execution of a prosthetic rehabilitation would have been extremely difficult because the amount of bone distal to the canine was insufficient to give proper support for an implant and mesial to the canine the mesio-distal space was inadequate. The plan deemed most effective was to move the canine distally to contact the first molar $(b, c)$ thus eliminating the posterior space. Later the canine would be reshaped to resemble a bicuspid. And at the same time sufficient additional space would have been created to make a highly favorable implant site in the maxillary canine area with its massive pillar of bone.

take advantage, as we did, of their healthier periodontal environment.

Implantology has brought important changes to orthodontic routines, forcing practitioners to think creatively about how to manage spaces around implant sites and how, in a multidisciplinary fashion, to conceive and to execute exotic treatment plans that were unheard of in the orthodox orthodontic world of only a few years ago.

This out-of-the-box thinking characterized our treatment plan preparation for the second patient who consulted us about the situation created by her loss many years ago of the upper right first and second premolars, which eventually resulted in the distal drift of the upper right canine into the place of the first bicuspid making a reconstruction scheme of joining it to the lateral impossible. In addition, the periodontal status made the placement of an implant between the canine and the first molar impractical (fig. 13 a to d).

We decided that the best course of action was a bold one, to move the canine distally into contact with the first molar thus simultaneously eliminating the distal edentulous area and enlarging the mesial edentulous area enough to make it a favorable site for an implant. The reconstruction of 16 , 13, and 13', a crown seated on an implant, made it possible for us to harmonize occlusal function. This pragmatic approach constituted, from both a therapeutic and financial point of view, a less costly procedure than the other possible solution of placing two implants in an area that would have required a considerable build-up of osseous tissue. 


\section{5 - CONCLUSION}

The periodontal environment is the most important factor for orthodontists to evaluate in their diagnostic assessments, particularly of adult patients, because it is the foundation upon which longevity of the dentition is based. Orthodontists understandably neglect to give careful consideration to this feature in planning treatment for adolescent patients because the vast majority of teen-agers have healthy periodontal tissues. Perhaps, in the future they would be well advised to pay more attention to this important facet of oral health. But for adults and for young children just forming their dentitions an evaluation of gingival status is essential. In the child, orthodontists may discern problems of teeth erupting outside the correct keratinized sites and have these potential trouble spots eliminated with muco-gingival surgery to assure tooth eruption in the most favorable conditions. In adults this assessment procedure guides orthodontists in making the best choices from a variety of possibilities, some of which may be unusual, that are adapted to the patient's particular needs and individualized to suit the patient's unique periodontal environment. The carefully selected treatment plan should not only promise a harmonious and equilibrated result but also, most important, one that will assure the longevity of the dentition.

\section{BIBLIOGRAPHY}

1. Bassigny F, Chabre C. Parodonte et Orthodontie chez le patient au cours de sa croissance. Orthod Fr 1984;55:203-349.

2. Bimstein E, Eidelman E. Morphological changs in the attached ans keratinized gengiva an gingival sulcus in the mixea dentition period after 5 years; longitudinal study. J Clin Periodontal 1988;15:175-9.

3. Bimstein E, Matcheti E, Becker A. The attached gingival in children: diagnosis, développemental and orthodontic considération for treatment. J Dent Child 1988;5:351-6.

4. Borghetti A, Monnet-Corti V. Chirurgie plastique parodontale. Paris: Cdp, 2008.

5. Fontenelle A. Une conception parodontale du mouvement dentaire provoqué: évidences cliniques. Rev Orthop Dento Faciale 1982;16:37-53.

6. Korbendau JM, Guyomard F. Chirurgie muco-gingivale chez l'enfant et I'adolescent. Paris: Cdp, 1992.

7. Laurelle L, Rylander H, Surdin Y. Histologic characteristics of clinically healthy gingiva in adolescents. Scand J Dent Res 1987;95:456-62.

8. Lüllmann-Rauch R. Histologie. Bruxelles: Edition de Boeck Université, 2008.

9. Maynard JG, Wilson RD. Diagnosis ans management of mucogingival problems in children. Deutclin North ARA 1980;24:683-703.

10. Melsen B. Place de I'orthodontie dans les traitements interdisciplinaires. Orthod $\mathrm{Fr}$ 1997;1:121-38.

11. Mombellé A., Gusberti FA, Oosten MC, Lang NP. Gingival health and jugivitis development durin puberty. A 4 year longitudival study. J Clinic Periodontal 1989;16:451-6. 
12. Oschenbein C, Maynard JG. The problems of attached gingiva in children. J Dent Clin 1974;41:263-72.

13. Schmid MO. The subperiostal vestibule extension. Litteratures review, rational and technique, J West SOC Periodont 1976;24:88-99.

14. Tenenbaum $H$, Tenenbaum M. A clinical study of the width of attached gengiva in the deciduous and permanent dentition. J Clin Parondontical 1986;13:270-5.

15. Timmerman MF, van der Weijden GA, Arief EM, Armand S, Abbas F, Winkel EG. Untreated periodontal desease in Indonesian adolescents. Subgingival microbiota in relation to experienced progression of periodonticis J Clin periodontal 2008;28:617-27. 